\title{
The Application of Spatial Signature Analysis to Electrical Test Data: Validation Study
}

\author{
Thomas P. Karnowski ${ }^{\mathbf{a}^{*}}$, Kenneth W. Tobin ${ }^{\mathrm{a}}$, Shaun S. Gleason ${ }^{\mathrm{a}}$, and Fred Lakhani ${ }^{\mathrm{b}}$ \\ ${ }^{a}$ Oak Ridge National Laboratory ${ }^{* *}$, P.O. Box 2008, Oak Ridge, Tennessee 37831-6011 \\ ${ }^{b}$ SEMATECH, 2706 Montopolis Drive, Austin, Texas 78741-6499
}

\section{ABSTRACT}

This paper presents the results of the Spatial Signature Analysis (SSA) Electrical-test (e-test) validation study that was conducted between February and June, 1998. SSA is an automated procedure developed by researchers at the Oak Ridge National Laboratory to address the issue of intelligent data reduction while providing feedback on current manufacturing processes. SSA was initially developed to automate the analysis of optical defect data. Optical defects can form groups, or clusters, which may have a distinct shape. These patterns can reveal information about the manufacturing process. Optical defect SSA uses image processing algorithms and a classifier system to interpret and identify these patterns, or "signatures". SSA has been extended to analyze and interpret electrical test data. The algorithms used for optical defect SSA have been adapted and applied to e-test binmaps. An image of the binmap is created, and features such as geometric and invariant moments are extracted and presented to a pair-wise, fuzzy, k-NN classifier. The classifier itself was prepared by manually training, which consists of storing example signatures of interest in a library, then executing an automated process which treats the examples as prototype signatures. The training process includes a procedure for automatically determining which features are most relevant to each class. The evaluation was performed by installing the SSA software as a batch process at three SEMATECH member company sites. Feedback from member company representatives was incorporated and classifiers were built to automatically assign labels to the binmap signatures. The three sites produced memory devices (DRAM) and microprocessors in a mature process fabrication environment. For all of these products, 5,620 signatures that encompassed approximately 552 wafers were human-classified and analyzed. The performance of the SSA E-test system indicates that the approach was successful in reliably classifying binmap signatures in a manner similar to the human expert.

Keywords: wafermap analysis, process signatures, automation, electrical test, yield learning, image processing

\section{INTRODUCTION}

As semiconductor device density and wafer area continue to increase, the volume of in-line and off-line data required to diagnose yield-limiting conditions is growing exponentially [1]. To manage this data in the future, analysis tools will be required that can automatically reduce this data to useful information, e.g., by assisting the engineer in rapid root-cause diagnosis of defect generating mechanisms. In this report, we describe a technology known as Spatial Signature Analysis (SSA) and its application to electrical test binmap data. SSA is an automated procedure that has been developed by researchers at the Oak Ridge National Laboratory to address the issue of intelligent data reduction while providing timely feedback on current manufacturing processes [1]. SSA was initially developed to automate the analysis of defect data collected by in-line microscopy and laser scattering systems [2]. A successful validation of this optical-based SSA approach was performed in the Spring of 1997 and reported through SEMATECH [3]. This method has been extended to analyze and interpret electrical test data and to provide a pathway for correlation of this data with in-line optical measurements [4]. The image processing-based, fuzzy classifier system used for optical defect SSA has been adapted and applied to e-test binmaps to interpret and rapidly identify characteristic patterns, or "signatures", in the binmap data that are uniquely associated with the manufacturing process. An image of the binmap is created, and features such as mass, geometric moments, and invariant moments are extracted and presented to a pair-wise, fuzzy, $k-N N$ classifier [5].

\section{SSA OVERVIEW}

Optical inspection of semiconductor wafers has long been the primary means of investigating the sources of wafer defects. Semiconductor yield engineers use high-resolution images of individual defects collected off-line to assess problems in the manufacturing process. Since high-resolution off-line defect review is time consuming and expensive, process engineers also

\footnotetext{
T.P.K. (Correspondence): Email: karnowskitp@ornl.gov; Telephone: 423-574-5732; FAX 423-574-6663

"* Prepared by OAK RIDGE NATIONAL LABORATORY, Oak Ridge, Tennessee, 37831-6285, managed by LOCKHEED MARTIN ENERGY RESEARCH COPR., for the U.S. DEPARTMENT OF ENERGY under contract DE-AC0596OR22464.
} 


\section{DISCLAIMER}

This report was prepared as an account of work sponsored by an agency of the United States Government. Neither the United States Government nor any agency thereof, nor any of their employees, make any warranty, express or implied, or assumes any legal liability or responsibility for the accuracy, completeness, or usefulness of any information, apparatus, product, or process disclosed, or represents that its use would not infringe privately owned rights. Reference herein to any specific commercial product, process, or service by trade name, trademark, manufacturer, or otherwise does not necessarily constitute or imply its endorsement, recommendation, or favoring by the United States Government or any agency thereof. The views and opinions of authors expressed herein do not necessarily state or reflect those of the United States Government or any agency thereof. 


\section{DISCLAIMER}

Portions of this document may be illegible in electronic image products. Images are produced from the best available original document. 
use low resolution defect wafermaps from in-line optical inspection tools to determine the potential source of problems in the manufacturing process. They accomplish this by analyzing and sourcing unique spatial distributions or "signatures" of defects on the wafer surface. A spatial signature is defined as a population of defects originating from a single manufacturing problem. Even when the optical spatial signatures do not contain significant portions of killer defects (i.e., defects that result in electrical faults), they do provide a diagnostic window into the manufacturing process. SSA attempts to emulate this process to provide the fabrication engineer with faster time-to-results, a critical requirement for effective yield learning and yield management.

SSA automatically collects defects on a wafermap that come from a single manufacturing source. A user-trained classifier assigns a label that identifies the root problem. SSA begins the signature classification process by converting the electronic wafermap file into a gray-scale image where each pixel is assigned an intensity value according to the number of defects in the subtended area. Each pixel represents a first level clustering of the individual defects. Clusters of pixels, denoted as "objects", in this density image are connected into multi-element objects (e.g., a multi-element scratch) by means of an advanced clustering procedure. Objects are grouped into high-level "sets" depending on their proximity to neighboring clusters and on their morphology[2, 6, 7].

Once an object has been assigned to a high-level set and characterized, its features are sent to a classifier where a userdefined label is assigned to the result. For this work, a pair-wise fuzzy k-Nearest Neighbor ( $\mathrm{kNN})$ [8] approach has been adapted that uses a unique feature reduction procedure to optimize classifier performance [9]. For industrial pattern recognition problems where it is difficult to ascertain a statistical parameterization for the large variety of class types encountered, non-parametric classifiers such as nearest mean or kNN [10] apply well. Furthermore, in an industrial setting, it is often required that the classifier system begins to classify new data with few training examples. Also, over the period of time required to collect large sample sets, acceptable process variations can occur which confuse the boundaries between classes. The pair-wise fuzzy kNN classifier training set can readily be maintained over time (e.g., by including and excluding examples based on time and date), and can operate adequately with relatively few examples for each class [11].

The SSA approach has been adapted to analyze e-test data. Similar to optical defect wafermaps, e-test wafermaps in many cases contain characteristic patterns, or signatures, that give insight into the health of the semiconductor manufacturing process. E-test wafermaps are commonly comprised of either bit data or bin data. Wafermaps created from bit data are called bitmaps, while wafermaps created from bin data are referred to as binmaps. Bit data result from performing electrical tests on the individual bits in a memory device, while bin data describe the results of electrical testing of individual die on a wafer. A bin can be viewed as a "bucket" classification into which all of the die that meet that classification fall. The most intuitive e-test bins are PASS and FAIL. Whether bit data or bin data are used, the e-test wafermap is created for viewing by "mapping" the results of these electrical tests onto a 2-D space. All wafer data used for this validation are binmap data. SSA Release 6.0 has the capability (at present) of analyzing only optical defect and e-test binmap data.

\section{Purpose}

\section{VALIDATION EXERCISE}

SSA research was initiated between SEMATECH and Oak Ridge in 1995. Initial development of the algorithms for signature segmentation and subsequent classification were based on early discussions with fabrication engineers and a broad spectrum of wafermap files donated to Oak Ridge by the various member companies. The optical wafermap validation exercise was performed in the Spring of 1997. We tested the maturity of the research and the SSA C++ software library in three different manufacturing environments on three separate products: ASIC, DRAM, and SRAM. The main goal of the 1997 validation was to prove the SSA technology in the fabrication environment for signature clustering and signature classification.

The main goal of the current exercise for e-test data was to prove the technology for binmap signature classification. Due to the nature of binmap data (i.e., a binmap image is composed of coarse die locations as opposed to high-resolution defect data as shown in Fig.1), the SSA algorithm was not used to break the binmap data into separate clusters. Instead, each signature is composed of the die which fail a particular bin code. The main goals of the validation exercise were to:

- verify the signature classification process for final test, binmap wafer data in the fab environment,

- better understand the application of SSA to final test data and the differences in needs expressed by the defect analyst and the final test analyst, 
- understand issues associated with integration of the analysis method into the fabrication environment and the plant yield management system.



(a)

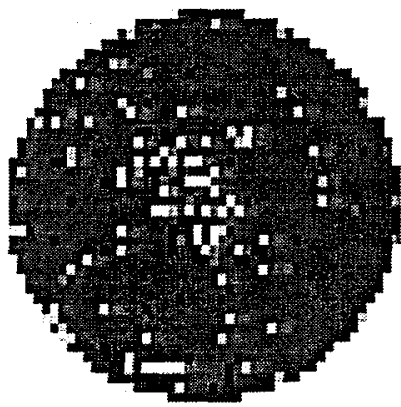

(b)

Figure 1 (a) High resolution wafermap showing a spin-coater "streak" pattern. (b) Final test binmap showing die (intensity coded) for each bin with a centrally heavy population of failed die.

\section{Site Selection}

Site selection was initiated in December, 1997, by sending out an SSA Validation Statement of Work (SOW) requesting MC participation in the project. Three sites were selected for participation. Due to the proprietary nature of the data collected at these three sites, they will remain anonymous and uncorrelated to the site labels and the data to be described below. Also, specific information that could be used to derive or infer site-specific product yield has been intentionally omitted. Figure 2 shows the representative characteristics of the three sites in relation to the well-known yield curve. The yield curve represents various levels of maturity in the manufacturing life cycle of a semiconductor device/product.

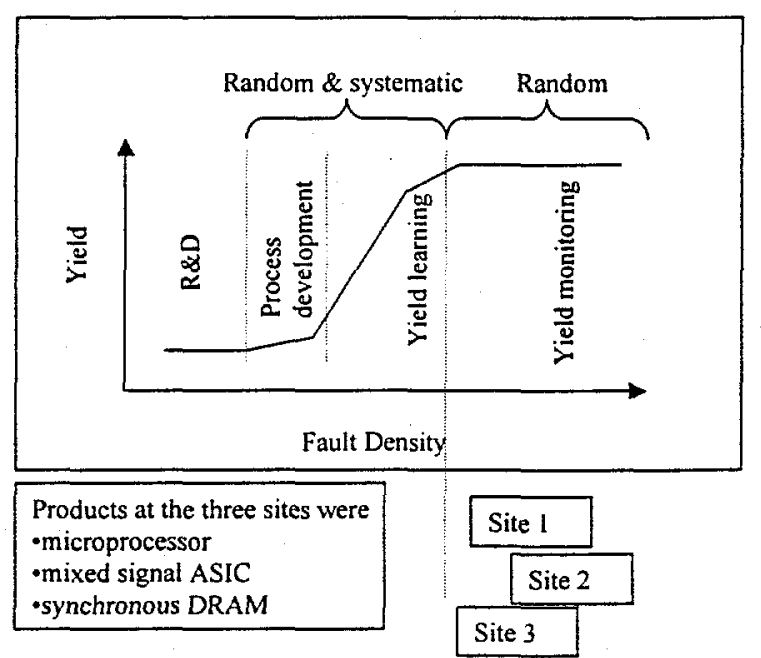

\section{Figure 2 Representative characteristics of the three participating MC sites relative to the yield curve.}

SSA technology has application to most phases of process development and product manufacturing. The left-most region of the curve is representative of the exploratory $R \& D$ phase. In this phase of process development, there are very few devices manufactured and yield is not the driving issue. At this stage, very little automation is required for data analysis. As the exploratory phase moves into the process development phase, higher wafer volumes necessitate the initiation of automation procedures to monitor a significant fraction of product. During the process development stage, systematic events are the dominant source of concern and most prevalent. Systematic events include mechanical wafer handling damage, plasma etch 
damage from arcing, systematic particle contamination during chemical vapor deposition, spin-on material contamination (e.g., glass, resist), and many other possible process related scenarios.

As the process development cycle begins to mature, it progresses to the yield learning phase. It is during this phase that the yield engineer will attempt to determine root-cause sources of systematic events and quickly eliminate them to increase yield. During this phase the number of wafers processed increases dramatically and SSA has the potential to very quickly assist the fabrication engineer in locating and correcting a large variety of problems in the manufacturing process. The yield learning ramp is of critical importance to the manufacturer. The rate at which yield learning is achieved is critical to the realization of a return on investment. By analyzing the maximum number of wafermaps with SSA during this stage, it is anticipated that yield learning can proceed much more quickly and that yield-impacting events can be more efficiently localized and corrected.

As the process matures over time, it enters the yield-monitoring phase. At this point the process technology is mature and acceptable device yields are maintained. A very small percentage of yield loss is related to systematic events for the controlled process and yield is primarily limited by random events, e.g., particles generated within process tools, or those originating from process gases and chemicals. At this phase, wafer volume is high and systematic yield loss is hard to detect. While SSA provides its main analysis advantage by monitoring single wafers during the yield learning phase, it will provide continued automatic capabilities during the yield monitoring phase by detecting subtle systematic events by overlaying multiple wafers together over single or multiple lots. It can also be used to monitor population statistics of random events. All three participating MC sites for the evaluation were operating in the yield-monitoring region of the curve.

\section{Software}

To support a concurrent validation effort at three manufacturing sites, it was necessary to develop a background batch SSA E-test software system that was capable of automatically processing and reporting on binmaps. It was also necessary that a viewer be developed which allowed the participants to document their comments on the efficacy of the procedure for the correct classification of events. This was accomplished in two steps as described below.


Figure 3 Schematic representation of the ssawatch batch processing system that automatically processed binmap files and e- mailed daily summaries to the participants.

Figure 3 shows a schematic representation of the background processor that was developed denoted ssawatch. The software ran continuously in the background on a Sun workstation and would periodically check for the arrival of new binmap files coming from the in-line inspection tools. As new binmap files arrived in the directory, they were processed and the SSA results were placed in a destination directory. On start-up, the ssawatch program took a resource file as input which established critical timing parameters such as where and when to check for new maps, which SSA electronic signature library (ESL) file to use, and when and where to send e-mail summaries for process results from the previous period (typically every 
12 hours. Note that the ESL contains labeled training samples of the various binmap signatures along with other classifier parameters. These files were generally unique to each site.

While this process ran continuously without the need for human intervention, it was required that the SSA results files be periodically reviewed by the participant on a regular basis for commentary. As mentioned above, this commentary was used to build and train an ESL file. Figure 4 shows the next step in the validation process. After the SSA-processed binmap files were placed in a results area (WATCH_DEST_VALID_INPUT_DIR in Fig. 3 and Fig. 4), about once or twice a week the user would run a separate interface tool called etest-validation that provided them with a convenient interface for viewing the wafermaps. The display tool showed the results file as a series of bin images with classification information. The user could opt to comment on the signatures or to move the file to a "no comment" area. Usually if a wafer had a normal or typical pattern of defects, no comment would be necessary, yet it was requested that all maps from the selected manufacturing process be sent to Oak Ridge for evaluation. Approximately once per week, the participant would log into a private account established on the Oak Ridge network and deposit their commented and uncommented binmaps for further analysis. These binmaps were used to develop and update an appropriate ESL for the different sites, and to perform a statistical analysis of the SSA performance.



Figure 4 Once the ssawatch software processed the binmaps, the user would supply comments on the maps using the etest-validation tool prior to sending the data to ORNL

\section{Method}

The validation statistics that are detailed in Section 5, Results, were generated from the commented and uncommented wafermap files sent to Oak Ridge from the three sites during the course of the validation. A program was written that would read all of the result files in a specified directory and output a flat ASCII file containing the various summary information needed to determine SSA performance.

Once the ASCII data file was obtained it was read into a spreadsheet. In the spreadsheet format, the data could easily be sorted for further analysis according to different parameters of interest. This analysis included the writing of a macro for generating confusion matrices as shown in Fig. 5. The confusion matrix gives a great deal of information regarding the classification of defects in a simple matrix format [10]. As shown in the figure, rows represent the actual class of the data (the " $i$ " index in the equations), i.e., as classified by the user (note that the user-classified data also contains classification error which is not folded into this analysis). The columns of the array represent the SSA-assigned class (the " $\mathrm{j}$ " index in the equations). By summing across a row, the actual number of samples of a particular class are ascertained. The diagonal elements of the matrix contains the number of signatures for each class that have been correctly classified by SSA. Therefore, the ratio of the diagonal element to the sum of the row gives an estimate of the performance of the classifier for that particular class. 


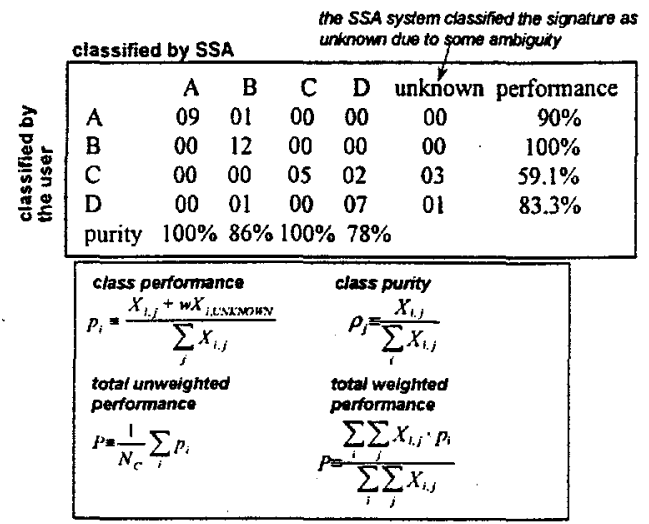

Figure 5 Example confusion matrix used to describe the statistical performance of the classifier.

The purity of a SSA classification decision is another indicator of the efficacy of the method. Purity is determined for a class by taking the ratio of the diagonal element to the sum of the column. While performance is a measure of how often a given class was identified correctly, purity is a measure of how often the identification of a given class was correct.

\section{Summary}

\section{RESULTS}

Table 1 gives a summary of classifier performance at the three manufacturing sites. There are two values of performance reported in the table: "total weighted performance" and "total unweighted performance". The first of these measures is weighted by the number of actual samples collected for each class. This metric allows the signatures of highest occurrence to carry the most weight in the performance estimate. The later measure assumes equal weight for each class. This is more indicative of performance assuming that every defined signature type is of equal importance. The metric that is "most correct", or most representative of the system performance, depends on the point of view of the reader. For this reason, both metrics are reported.

Table 1 - Summary of SSA E-test classifier performance for the three participating MC sites determined during the validation exercise.

\begin{tabular}{|l|c|c|c|}
\hline & SITE 1 & SITE 2 & SITE 3 \\
\hline $\begin{array}{l}\text { Total Weighted } \\
\text { Performance }\end{array}$ & $\mathbf{7 9 \%}$ & $\mathbf{8 4 \%}$ & $\mathbf{7 2 \%}$ \\
\hline $\begin{array}{l}\text { Total Unweighted } \\
\text { Performance }\end{array}$ & $\mathbf{7 4 \%}$ & $\mathbf{8 1 \%}$ & $\mathbf{6 4 \%}$ \\
\hline Average Purity & $69 \%$ & $80 \%$ & $66 \%$ \\
\hline No. of Defined Classes & 9 & 15 & 11 \\
\hline $\begin{array}{l}\text { No. of Signatures (Bins) } \\
\text { Analyzed }\end{array}$ & 2004 & 2105 & 1511 \\
\hline $\begin{array}{l}\text { Average No. of Bins per } \\
\text { Wafer }\end{array}$ & 7 & 30 & 7 \\
\hline Approximate No. of Wafers & 286 & 70 & 196 \\
\hline
\end{tabular}




\section{Training Data}

The SSA E-test system is trained using a small sample population of signatures. The training library typically contains 5 to 15 examples from each class defined by the user for their site. The system performs a training optimization procedure that provides useful feedback to the operator on the efficacy of their class definitions and the examples that they placed in each category. Figure 6 shows the final training results from each of the three sites. The top region of the figure, (a), shows the user-defined class labels followed by the number of examples that were selected for each category. The second region, (b), contains the credit, hold-one-out (HOO) performance, and the resulting defuzzification value. The credit is the amount of user credit given to the classifier whenever classification of "unknown" is assigned to a data sample. The "unknown" designation means that the classifier does not have enough confidence to assign a crisp class to the particular sample. The HOO performance is also known as the expected performance and it is an estimate of how well the trained classifier will perform in the field when exposed to new data. Our experience has been the actual field performance is slightly lower than the expected performance in most instances. This is due to the fact that samples are collected for training over a short period

\begin{tabular}{|c|c|c|c|}
\hline 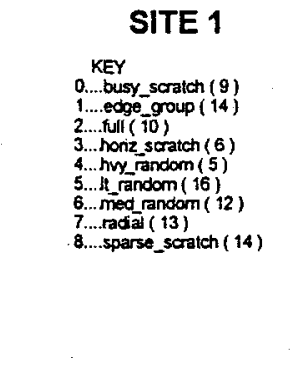 & 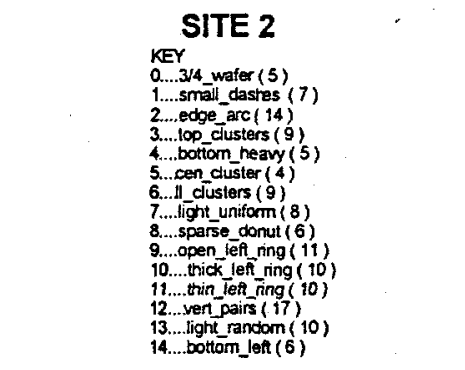 & 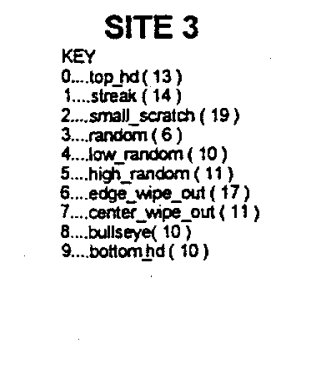 & $a$ \\
\hline $\begin{array}{l}\text { Credit far unknown: } 0.3 \\
\text { Pertormance from HOO: } 0.730303 \\
\text { Defurzification value } 0.85\end{array}$ & $\begin{array}{l}\text { Creofit for unknown: } 0.3 \\
\text { Performance frtam } 1000: 0.933588 \\
\text { Defuzzification value } 0.92\end{array}$ & $\begin{array}{l}\text { Credit for uniknown: } 0.3 \\
\text { Pertormance from } H O O: 0.8 \\
\text { Defuzzification value } 0.89\end{array}$ & \\
\hline 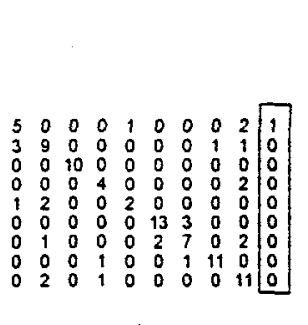 & 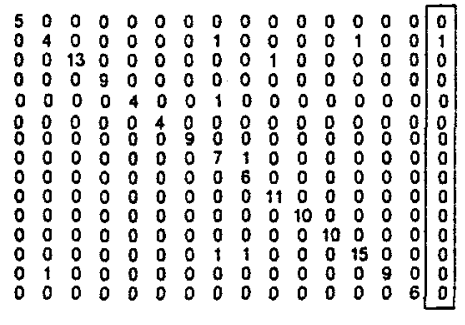 & 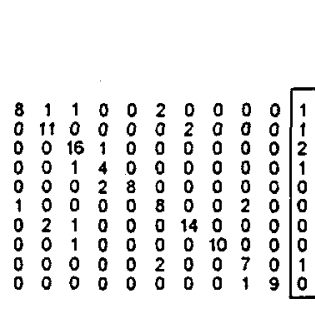 & C \\
\hline
\end{tabular}

\section{Figure 6 Results of training the E-test classifier for validation sites. (a) Labels assigned and the number of examples. (b) Hold-one-out (HOO) data (c) confusion matrix.}

of time while field use takes place over a longer period thus allowing for some drift in the nature (e.g., morphology) of the signature data. The final value is the defuzzification threshold. When the SSA fuzzy classifier assigns a crisp label to the signature, the signature's membership value must be greater than the defuzzification threshold, otherwise the signature is assigned to the "unknown" category.

The final region, (c), contains the confusion matrix for the HOO classifier optimization. The right-most column in the confusion matrix contains the number of training samples that the classifier did not have confidence in labeling during training.

The following figures illustrate details for validation testing at each site. Figure 7 graphically shows the signature categories collected at each site for the user-defined classes. Each row in the figure represents a user-defined class of data. The goal of including these signature figures is to give the reader an indication of the variety of classes defined by the validation participants during testing. Table 2 gives the confusion matrix for all hand-labeled test data collected at Site 1 . Figures 8 and 9 and Tables 3 and 4 shows similar detail for validation Sites 2 and 3 respectively. 




Figure 7 - Image of the user-defined classes selected for Site 1. Each row corresponds to a single user-defined class.

Table 2 - Confusion matrix generated from the 2004 hand-labeled test signatures collected at Site 1.

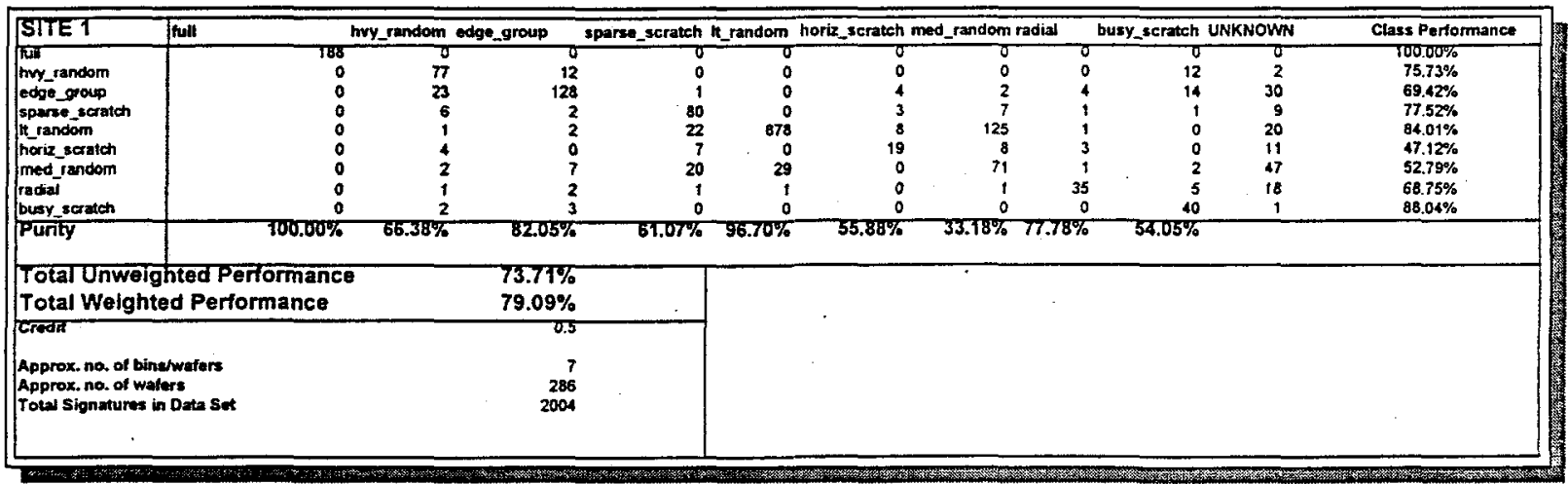






Figure 7 - Image of the user-defined classes selected for Site 2. Each row corresponds to a single user-defined class.

Table 3 - Confusion matrix generated from the $\mathbf{2 1 0 5}$ hand-labeled test signatures collected at Site 2.

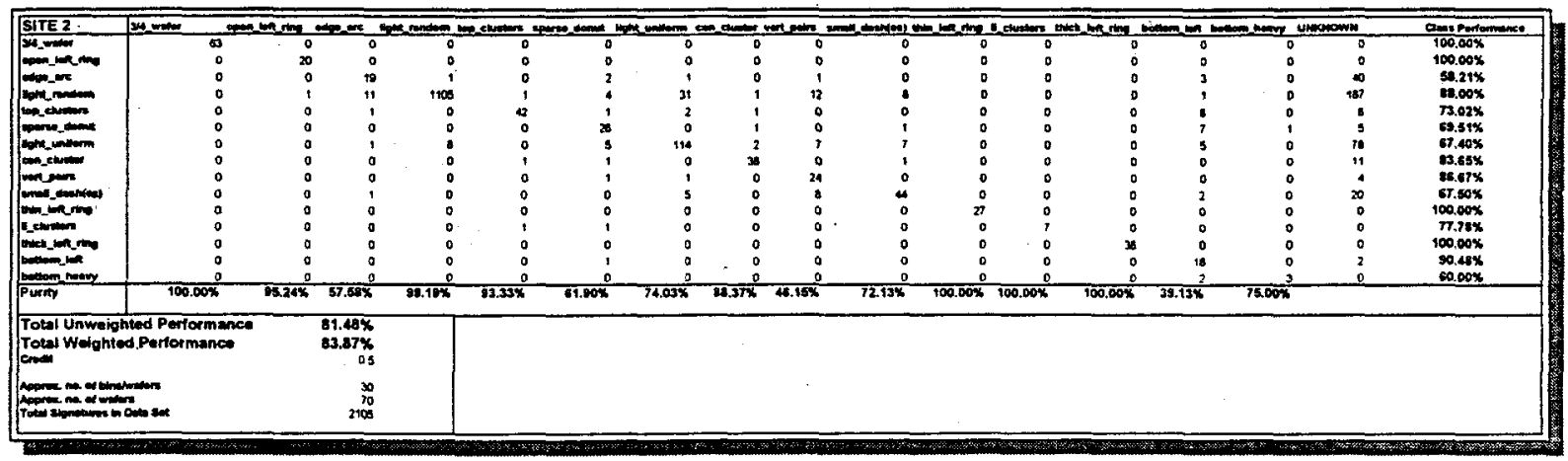




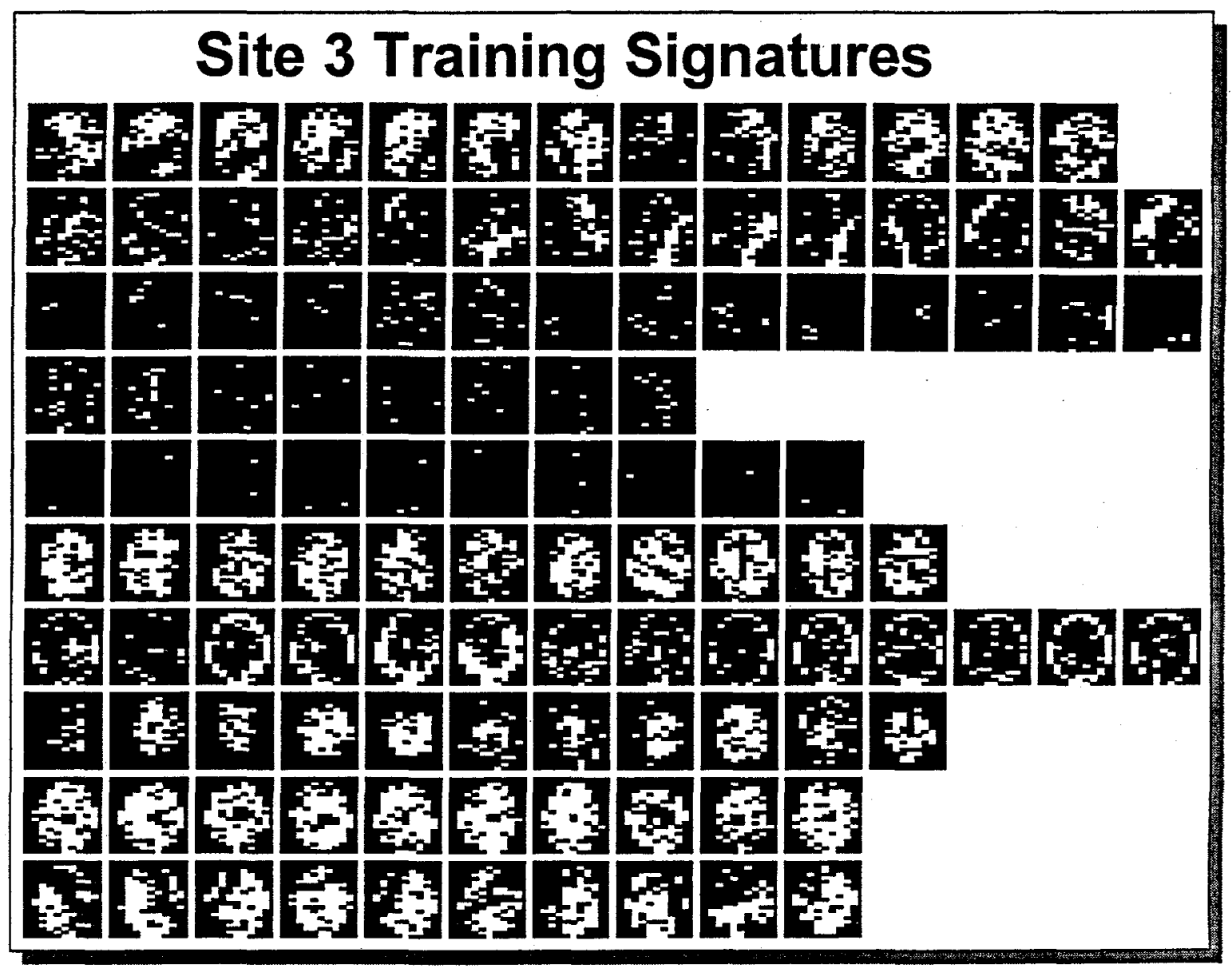

Figure 8 - Image of the user-defined classes selected for Site 3. Each row corresponds to a single user-defined class.

Table 4 - Confusion matrix generated from the 1511 hand-labeled test signatures collected at Site 3.

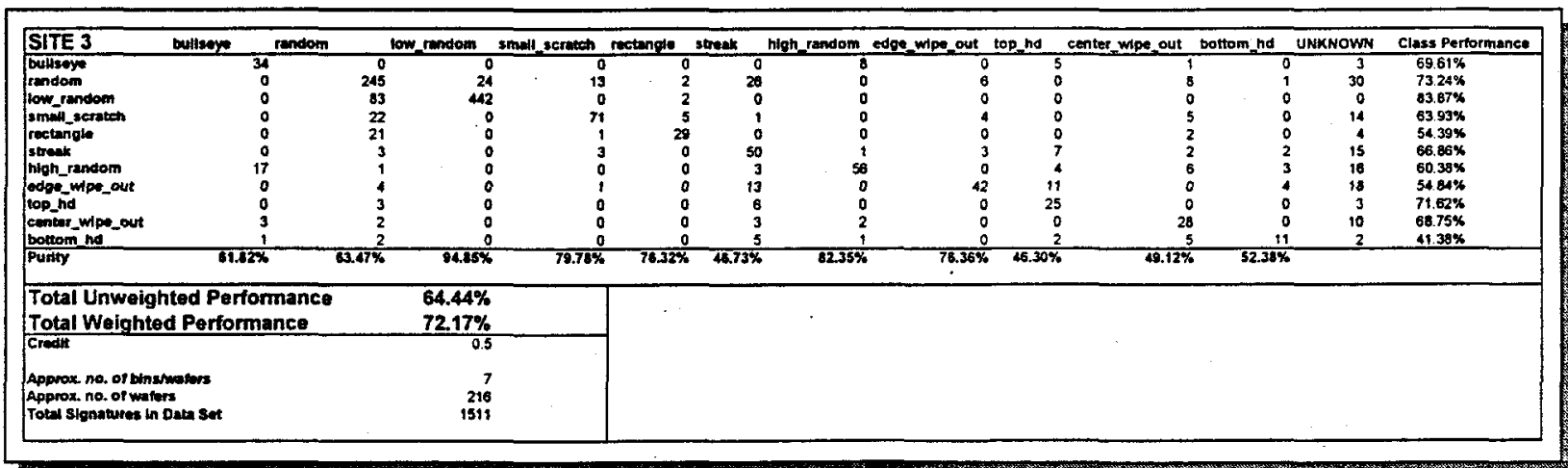




\section{Discussion}

The results presented in previous sections indicate an average overall system performance of $75 \%(70 \%)$. Generally, a human classification expert is approximately $70 \%$ reliable when classifying process defects and signatures. Since the classification data provided during this validation exercise has higher overall consistency than typical field data (due to the short-term and focussed nature of this work), it is asserted that the SSA system performs as well or slightly better than the average field engineer. In addition, the SSA E-test system provides the benefits of an automated computer vision application such as robustness, reliability, and continuous, uninterrupted operation of the system over extended periods of time.

The performance of the system at Site 3 deserves further discussion. The total performance reported for Site 3 was lower than either Site 1 or 2 . It is believed that the lower performance is related to two issues: (1) the coarseness of the bin patterns due to the fewer number of die per wafer, and (2) the means by which SSA-E-test considers clustered data. SSA is an image processing tool that relies on the image of the bin data to perform feature analysis. A wafer with few die will look to the system like a low-resolution image and therefore the feature descriptions will effectively be "blurred". This effect also folds into the second issue associated with clustering. Since e-test data is presented to SSA on the die-level, as opposed to defect data which has resolution on the order of the sub-die level, it was determined from early testing (prior to the field validation exercise) that it would be problematic to segment the wafermaps into smaller groupings, or clusters, of die patterns. Although this will be discussed further, the result for Site 3 data was to cause confusion between classes such as "small scratch" and "random" since a "small scratch", as defined by the user, could be contained in a "random" (or other) pattern.

Due to an apparent need to accommodate some level of clustering at all sites, a technique was adopted and integrated into the SSA system. A simple extension of the SSA E-test approach to accommodate some level of clustering was achieved by measuring binmap features as follows: a measurement of features from the whole wafer was performed; next, a measurement of features from the largest connected group of dies was performed; and finally, a measure of the wafers features was performed when all single, unconnected die were removed. This extended list of features was used to describe the wafer in the classification process.

At least two of the test site participants had made classification comments on wafers that were related to the particular failed bin code. For example, some signatures had one meaning when they had a certain bin code and different definitions when in another bin. SSA E-test does not currently take into account a bin-dependent classification method. A similar occurrence occurred during optical defect SSA validation where a user wanted to classify a pattern based on the process layer. This was easily implemented in the commercial version of SSA by allowing a layer-dependent library to be created by the user. This same approach can be taken to accommodate a bin-specific library in future versions of SSA E-test in the manufacturing environment.

The elemental set approach used for optical defect SSA allowed the classification problem to be defined as four simpler problems, and therefore the system could run more efficiently and be trained fairly easily (the elemental grouping of the data was performed transparently to the user). With SSA E-test, there is only one classifier that is developed by the user. It was noted during this testing that the definition of a signature library was more difficult than in optical defect SSA. This was likely due to several issues. The inability to place signature examples into different clustered categories (due to the inherent coarseness of binmap data) made it difficult for the user to logically separate some signature types into unique, well-defined groups. Also, the training method for the SSA E-test system provides feedback to the user on how well the classifier is expected to perform in the field (i.e., when exposed to data not seen prior to training). To achieve good expected performance during training, it was typically required that the user reduce, or simplify, the number of defined classes, i.e., good performance could sometimes be achieved only at the cost of good class resolution. Finally, the lack of several elemental categories can result in a signature library that contains many classes and training examples. A more complex classification library can result in longer processing times and more complexity in the decision-making process.

\section{CONCLUSIONS}

The SSA E-test validation exercise was able to successfully demonstrate the concepts and issues associated with the automatic classification of e-test, binmap data. The overall reported efficacy of $75 \%(70 \%)$ is very encouraging and shows that the system can work in the manufacturing environment to the level of its human counterpart for a well-defined set of binmap signature classes. It is hoped that the data presented in this report will prove useful to those licensees of the SSA Defect and E-test system and will provide them with a fair and accurate understanding of both the benefits and limitations of the technology. 


\section{REFERENCES}

1 K.W. Tobin, S.S. Gleason, F. Lakhani, and M.H. Bennett, "Automated Analysis for Rapid Defect Sourcing and Yield Learning", Future Fab International, Issue 4, Vol. 1, Technology Publishing Ltd., London 1997, p. 313.

2 S. S. Gleason, K. W. Tobin, and T. P. Karnowski, "Spatial Signature Analysis of Semiconductor Defects for Manufacturing Problem Diagnosis", Solid State Technology, July, 1996.

3 K. W. Tobin, S. S. Gleason, T. P. Karnowski, M. H. Bennett, "An Image Paradigm for Semiconductor Defect Data Reduction", SPIE's 1996 International Symposium on Microlithography, Santa Clara Convention Center, Santa Clara, CA, March 10-15, 1996.

4 K.W. Tobin, S.S. Gleason, T.P. Karnowski, S.L. Cohen, and F. Lakhani, "Semiconductor Spatial Signature Analysis: Validation and Status Report", SEMATECH Technology Transfer No. 97073322A-TR, Austin, Texas, August 30, 1997.

5 S.S. Gleason, K.W. Tobin, T.P. Karnowski, and F. Lakhani, "Rapid Yield Learning through Optical Defect and Electrical Test Analysis", SPIE's 1997 International Symposium on Microlithography, Santa Clara Convention Center, Santa Clara, CA, February, 1997.

6 Gleason, S.S., and Tobin, K.W., "Directional-based Dilation for Connection of Piece-Wise Objects: A Semiconductor Manufacturing Case Study," International Conference on Image Processing, Lausanne, Switzerland, Sept 16-19, 1996.

7 T.P. Karnowski, S.S. Gleason, K.W. Tobin, "Fuzzy Connectivity of Semiconductor Defect Events," SPIE Proceedings on Machine Vision Applications in Industrial Inspection, January, 1998.

8 J. M. Keller, M. R. Gray, and J. A. Givens, Jr., "A Fuzzy K-Nearest Neighbor Algorithm," IEEE Transactions on Systems, Man, and Cybernetics, Vol. SMC-15, No. 4, p. 580, July/August 1985.

9 K.W. Tobin, S.S. Gleason, and T.P. Karnowski, "Adaptation of the Fuzzy K-Nearest Neighbor Classifier for Manufacturing Automation", SPIE's 10th International Symposium on Electronic Imaging: Science and Technology, San Jose Convention Center, January 1998.

10 R. O. Duda, and P. E. Hart, Pattern Classification and Scene Analysis, John Wiley \& Sons, New York, 1973, p.95.

11 K.W. Tobin, S.S. Gleason, T.P. Karnowski, S.L. Cohen, and F. Lakhani, "Automatic Classification of Spatial Signatures on Semiconductor Wafermaps", SPIE's 22nd Annual International Symposium on Microlithography, Santa Clara Convention Center, Santa Clara, CA, March 9-14, 1997. 